\title{
Genetic association study of coronary collateral circulation in patients with coronary artery disease using 22 single nucleotide polymorphisms corresponding to 10 genes involved in postischemic neovascularization
}

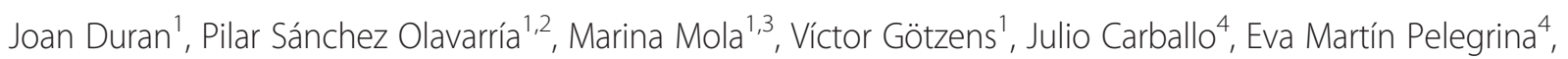
Màrius Petit ${ }^{4}$, Omar Abdul-Jawad ${ }^{6}$, Imanol Otaegui ${ }^{5}$, Bruno García del Blanco ${ }^{5}$, David García-Dorado ${ }^{5}$, Josep Reig ${ }^{7}$, Alex Cordero ${ }^{1,8}$ and Josep Maria de Anta ${ }^{1 *}$

\begin{abstract}
Background: Collateral growth in patients with coronary artery disease (CAD) is highly heterogeneous. Although multiple factors are thought to play a role in collateral development, the contribution of genetic factors to coronary collateral circulation (CCC) is largely unknown. The goal of this study was to assess whether functional single nucleotide polymorphisms (SNPs) in genes involved in vascular growth are associated with CCC.
\end{abstract}

Methods: 677 consecutive CAD patients were enrolled in the study and their CCC was assessed by the Rentrop method. 22 SNPs corresponding to 10 genes involved in postischemic neovascularization were genotyped and multivariate logistic regression models were adjusted using clinically relevant variables to estimate odds ratios and used to examine associations of allelic variants, genotypes and haplotypes with CCC.

Results: Statistical analysis showed that the HIF1A rs1 1549465 and rs2057482; VEGFA rs2010963, rs1570360, rs699947, rs3025039 and rs833061; KDR rs1870377, rs2305948 and rs2071559; CCL2 rs1024611, rs 1024610, rs2857657 and rs2857654; NOS3 rs1799983; ICAM1 rs5498 and rs3093030; TGFB1 rs1800469; CD53 rs6679497; POSTN rs3829365 and rs1028728; and LGALS2 rs7291467 polymorphisms, as well as their haplotype combinations, were not associated with CCC $(p<0.05)$.

Conclusions: We could not validate in our cohort the association of the NOS3 rs1799983, HIF1A rs11549465, VEGFA rs2010963 and rs699947, and LGALS2 rs7291467 variants with CCC reported by other authors. A validated SNP-based genome-wide association study is required to identify polymorphisms influencing CCC.

Keywords: Collateral circulation, Arteriogenesis, Postischemic neovascularization, Single nucleotide polymorphism, Genetic association study

\footnotetext{
* Correspondence: janta@ub.edu

${ }^{1}$ Unitat d'Anatomia i Embriologia Humanes, Departament de Patologia i

Terapèutica Experimental, Facultat de Medicina, Campus de Ciències de la

Salut de Bellvitge, Universitat de Barcelona, L'Hospitalet de Llobregat,

Barcelona 08907, Spain

Full list of author information is available at the end of the article
} 


\section{Background}

In patients with coronary artery disease (CAD), the perfusion of the myocardial tissue is impaired. To mitigate myocardial ischemia, a neovascularization process, which includes the creation of a capillary network in the ischemic myocardium (angiogenesis) and the growth of collateral arteries (arteriogenesis) is initiated to enhance blood flow to the myocardium. Collateral arteries are natural vascular bypasses that can significantly reduce the degree of myocardial ischemia. They develop through the growth of small pre-existing arterioles [1]. Thus, patients with good collateral circulation have a lower mortality (36 \%) than patients with low levels of collateralization [1]. Patients with $\mathrm{CAD}$ are highly heterogeneous in their arteriogenic response, even those with totally occluded arteries [2], with this variability attributed to genetic and environmental factors [3]. Collateral vascular growth and angiogenesis are parts of the same process leading to neovascularization. They complement each other: collateral growth and arteriogenesis provide bulk flow to the tissue, and angiogenesis promotes a capillary network that salvages the ischemic area. Angiogenesis and arteriogenesis are driven by distinct, but partially overlapping, cellular and molecular pathways [4]. In this study we examine putative genetic markers of coronary collateral growth. Our group has previously reported that the p.Pro141Leu polymorphism located in the urokinase-type plasminogen activator gene (PLAU), a gene expressed at collateral growth sites during arteriogenesis, is associated with coronary collateral development in patients with severe CAD [5]. To this end, we performed an association study to relate coronary collateral circulation (CCC) to 22 SNPs corresponding to 10 genes with suspected or demonstrated functional involvement in the process of postischemic neovascularization, and their corresponding haplotypes, in a Spanish cohort of patients with CAD.

\section{Methods}

\section{Study subjects}

The study was conducted between 2008 and 2012. We evaluated a Spanish cohort of 677 consecutive CAD patients with severe $(\geq 70 \%)$ stenosis who had been scheduled to undergo diagnostic coronary angiography at the Centre Cardiovascular Sant Jordi (CCSJ) or the Hospital Universitari Vall d'Hebron (HUVH) in Barcelona, Spain. The protocol was approved by the Bioethics Committee of the two centers (Ethics Committee of Clinical Research of the HUVH and the Bioethics Committee of the CCSJ), and authorized written consent was obtained from all the subjects. The exclusion criteria were: recent (less than 1 month previously) acute myocardial infarction; anemia; recent angioplasty; prior revascularization by percutaneous coronary intervention; coronary artery bypass surgery; and renal infection, inflammation or chronic failure. Epidemiological and clinical data included hypertension, diabetes mellitus (DM), DM type, hyperlipidemia, smoking history, family history of cardiopathies (FHC), history of angina, angina type and acute myocardial infarctions (AMI); with those not referring to type recorded as present or absent.

\section{Coronary angiography and coronary collateral artery scoring}

Selective coronary angiography was performed using multiple orthogonal projections via the Judkins technique. Injection of the contrast in the donor artery was performed at a sustained high pressure with an automated controlled machine (ACIST CVi Contrast Delivery System ${ }^{\circ}$ ). CCC was assessed angiographically using a "modified" Rentrop's method [6] without occlusion of the recipient artery. The following scale was used to assess the level of filling of the channels: $0=$ no visible filling of any collateral channels; $1=$ collateral filling of branches of the vessel to be dilated without any dye reaching the epicardial segment of that vessel (that is, right coronary artery injection showing retrograde filling of septal branches to their origin from the left anterior descending artery, without visualization of the latter occluded artery); 2 = partial collateral filling of the epicardial segment of the vessel being dilated; and $3=$ complete collateral filling of the vessel being dilated. In patients with more than one collateral vessel, the highest Rentrop score was recorded.

CAD patients were classified according to the degree of CCC as either poor CCC (Rentrop $0-1)(n=546)$ or good CCC (Rentrop 2-3) $(n=131)$. CCC was assessed by three experienced cardiologists who were blinded to the epidemiological, clinical and genetic data. The degree of agreement in the evaluation of CCC was high among the 3 observers, as determined by the kappa coefficient: $\mathrm{K}=0.987 ; 95 \%$ confidence interval $(95 \% \mathrm{CI})$, $0.953-1.000(\mathrm{P}<0.001)$ using the first 100 angiograms.

\section{SNP selection and genotype analysis}

22 SNPs of genes involved in postischemic neovascularization were selected attending the following criteria: a) their suspected or proved functional or/and clinical significance regarding angiogenesis or arteriogenesis when known; b) their location within coding, $5^{\prime}$ or $3^{\prime}$ untranslated, or intronic sequences with known potential sites for factor binding; and c) a minor allele frequency of more than $5 \%$ in the population studied (NCBI). We searched genes directly or indirectly involved in angiogenesis and/or arteriogenesis containing functional polymorphisms. Particularly, HIF1A [7-9], VEGFA [10-12], KDR [13, 14], NOS3 [15, 16], TGFB1 [17-19] and LGALS2 [20, 21] have been involved in both processes. 
Furthermore, CCL2 [22] and ICAM1 [23] play an important role in arteriogenesis, while $C D 53$, which controls TNF $\alpha$ levels [24], also plays an important role in this process [25]; and POSTN has been reported to be involved in angiogenesis [26] (Table 1). The SNPs located in or near these genes that were analyzed in this study are listed in Table 1 and details of them are as follows. HIF1A rs11549465 and rs2057482 affect mRNA production and are associated with CAD [27]; the first is also related to collateral circulation [28]. VEGFA rs2010963, rs1570360 and rs699947 influence protein production [29], and along with rs3025039 and rs833061 they have also been related to VEGFA serum levels [30-32]. Moreover, VEGFA rs2010963 and rs699947 have been associated with collateral circulation [33] and CAD [34]. KDR rs1870377 and rs2305948 affect primary protein structure, whereas rs2071559 is located 5' upstream, being all related to CAD [35]. CCL2 rs1024611 affects mRNA production [36-38]; and along with rs1024610, MCP1 plasma levels [39-41]. CCL2 rs1024611 and rs1024610 have been associated with myocardial infarction [39, 42]. NOS3 rs1799983 has functional consequences for the protein $[43,44]$ which are associated with coronary arteriogenesis $[45,46]$ and CAD [47]. ICAM1 rs5498 affects the primary structure of the protein and both it and rs3093030, located near the 3' end of the gene, are related to sICAM1 plasma levels [48-51] and to coronary artery calcification [52]. TGFB1 rs1800469 is located towards the 5' end of the gene and has been associated with coronary heart disease complications [53]. CD53 rs6679497 is an intronic polymorphism associated with TNF $\alpha$ levels [24] which plays a role in modulating arteriogenesis [25]. POSTN rs3829365 and rs1028728 are located in the 5' UTR of the gene, with the first being associated with heart failure [54]. Finally, LGALS2 rs7291467 is located in intron 3 and has been associated with arteriogenesis [21] and CAD [55-57].

Blood samples were drawn from patients undergoing coronary artery catheterization. Genomic DNA was isolated using the QIAmp DNA Blood kit following the manufacturer's protocol (Qiagen $\odot$, UK). TaqMan SNP genotyping assays (Applied Biosystems, Foster City, CA, USA) were performed to determine genotypes from the blood samples using a 7900HT Fast Real-Time PCR System (Applied Biosystems, Foster City, CA, USA). Genotype assessments were reproduced in three independent assays.

\section{Statistical Analysis}

Data were summarized and presented in the form of mean, standard deviation and percentage as descriptive statistics. Continuous data that were not normaldistributed were analyzed using the Mann-Whitney $U$ test. In this study, age does not show a normal distribution (Shapiro-Wilk p-value $<0.001$ ). Associations among categorical data were assessed using Fisher's exact or chisquare test, and Hardy Weinberg equilibrium was assessed using the chi-square test. Multivariate logistic regression models were adjusted using clinically relevant variables to estimate odds ratios (ORs) and $95 \%$ CIs among genotypes, haplotypes and the risk of poor CCC. Interaction terms between SNPs, haplotypes and significant covariates were also analyzed in the multivariate regression models. Statistical analysis was performed using STATA 11.2 software. The power to detect a genetic association was estimated using the same statistical package. The SNPStats software available at http://bioinfo.iconcologia.net/en/SNPStats_ web was used to calculate linkage disequilibrium (measured as Lewontin's D0-values) between SNPs, to estimate haplotype frequencies, and to evaluate haplotype association with CCC.

\section{Results}

A total of 677 CAD patients (median of age 66 years, 107 females/570 males) stratified according to the level of coronary collateralization (546 poor; 131 good) were enrolled in the study. The clinical and epidemiological parameters of the patients according to CCC development are listed in Table 2. Statistical analysis showed that there were no differences among the poor and good CCC groups in terms of age, gender, hypertension or hyperlipidemia history, smoking, angina history or previous myocardial infarction (Table 2). However, the incidence of DM (55.9 \%) and the percentage of patients prescribed with statins ( $44.3 \%)$ were significantly higher in the poor CCC group, with $\mathrm{p}$ values of 0.037 and 0.035 respectively (Table 2).

None of the SNPs studied, with the exception of NOS3 rs1799983 and POSTN rs3829365, showed any deviation from Hardy-Weinberg equilibrium (HWE) (tested by conventional $X 2$ ) (Table 3). Therefore, rs1799983 $\left(\mathrm{P}_{\text {HWErs1799983 }}=0.0157\right)$ and rs3829365 $\left(\mathrm{P}_{\text {HWErs3829365 }}=\right.$ $0.0000)$ were not included in further genetic association tests.

The genotype and allele distributions of all the polymorphisms in the population studied are shown in Table 2, and they did not show any differences between patients with good collateralization and patients with poor collateralization $(\mathrm{p} \geq 0.05)$ (Table 3$)$. Haplotype association analysis of polymorphisms in strong LD has more power than single locus tests to detect gene-disease associations. Thus, we also checked for haplotype combinations of polymorphisms in the VEGFA, KDR, CCL2, ICAM1, and POSTN genes to detect associations with CCC. To this end, we first estimated LD between the polymorphisms of these genes. There was a strong pairwise LD between the SNPs within these genes (data not shown), and VEGFA, KDR, CCL2, ICAM1 and 
Table 1 SNPs analyzed in the study

\begin{tabular}{|c|c|c|c|c|c|c|c|c|c|}
\hline Gene & $\begin{array}{l}\text { Role in angiogenesis/ } \\
\text { arteriogenesis }\end{array}$ & SNP & $\begin{array}{l}\text { Other HGVS } \\
\text { names }\end{array}$ & Location & $\begin{array}{l}\text { Functional } \\
\text { category }\end{array}$ & FS score & $\begin{array}{l}\text { Association } \\
\text { to CCC }\end{array}$ & Association to CAD & Functional relevance \\
\hline \multirow[t]{2}{*}{ HIFTA } & Both $[7,8]$ & rs11549465 & p.Pro582Ser & Exon 2 & Missense variant & 0.627 & [28] & [27] & Influences transactivation activity $[27,58]$ \\
\hline & & rs2057482 & $c . * 45 T>C$ & $3^{\prime}-U T R$ & 3' UTR variant & 0 & - & [27] & Influences transactivation activity [27] \\
\hline \multirow[t]{5}{*}{ VEGFA } & Both [10-12] & rs2010963 & c. $-634 C>G$ & Promoter & $\begin{array}{l}\text { Regulatory region } \\
\text { variant }\end{array}$ & 0.257 & [33] & [34] & $\begin{array}{l}\text { Influences protein production [29] } \\
\text { Related to VEGFA serum levels [30] }\end{array}$ \\
\hline & & rs1570360 & $\begin{array}{l}\text { c. }-1154 A> \\
G\end{array}$ & Promoter & $\begin{array}{l}\text { Regulatory region } \\
\text { variant }\end{array}$ & 0.242 & - & - & $\begin{array}{l}\text { Influences protein production and } \\
\text { related to VEGFA serum levels [31] }\end{array}$ \\
\hline & & rs699947 & c. $-2055 \mathrm{~A}>\mathrm{C}$ & $\begin{array}{l}\text { Upstream } \\
\text { gene }\end{array}$ & $\begin{array}{l}\text { Regulatory region } \\
\text { variant }\end{array}$ & 0.176 & [33] & [34] & $\begin{array}{l}\text { Influences protein production and } \\
\text { related to VEGFA serum levels }[30,31]\end{array}$ \\
\hline & & rs3025039 & $c^{*} 237 C>T$ & $3^{\prime}-U T R$ & 3' UTR variant & 0 & - & - & Related to VEGFA serum levels [32] \\
\hline & & rs833061 & c. $-958 \mathrm{C}>\mathrm{T}$ & Promoter & $\begin{array}{l}\text { Regulatory region } \\
\text { variant }\end{array}$ & 0.282 & - & - & Related to VEGFA serum levels [30] \\
\hline \multirow[t]{3}{*}{$K D R$} & Both $[13,14]$ & rs1870377 & p.Gln472His & Exon 11 & Missense variant & 0.103 & - & [35] & - \\
\hline & & rs2305948 & p.Val297lle & Exon 7 & Missense variant & 0.621 & - & [35] & - \\
\hline & & rs2071559 & c. $-906 \mathrm{~T}>\mathrm{C}$ & $\begin{array}{l}\text { Promoter } \\
\text { flanking }\end{array}$ & $\begin{array}{l}\text { Regulatory region } \\
\text { variant }\end{array}$ & & - & [35] & - \\
\hline \multirow[t]{4}{*}{ CCL2 } & Arteriogenesis [22] & rs1024611 & g. $2493 A>G$ & $\begin{array}{l}\text { Promoter } \\
\text { flanking }\end{array}$ & $\begin{array}{l}\text { Regulatory region } \\
\text { variant }\end{array}$ & 0.208 & - & Related to myocardial infarction $[39,42]$ & $\begin{array}{l}\text { Related to MCP1 serum levels [39-41] } \\
\text { Influences mRNA expression [36-38] }\end{array}$ \\
\hline & & rs 1024610 & g.2936 T > A & $\begin{array}{l}\text { Promoter } \\
\text { flanking }\end{array}$ & $\begin{array}{l}\text { Regulatory region } \\
\text { variant }\end{array}$ & 0.158 & - & Related to myocardial infarction [39] & Related to MCP1 serum levels [39] \\
\hline & & rs2857657 & g. $5837 \mathrm{G}>\mathrm{C}$ & $\begin{array}{l}\text { Non } \\
\text { coding } \\
\text { exon }\end{array}$ & $\begin{array}{l}\text { Non coding } \\
\text { transcript exon } \\
\text { variant }\end{array}$ & 0.176 & - & - & - \\
\hline & & rs2857654 & g. $2236 C>$ A & $\begin{array}{l}\text { Promoter } \\
\text { flanking }\end{array}$ & $\begin{array}{l}\text { Regulatory region } \\
\text { variant }\end{array}$ & 0 & - & - & - \\
\hline NOS3 & Both $[15,16]$ & rs1799983 & p.Asp298Glu & Exon 7 & Missense variant & 1 & {$[45,46]$} & [47] & $\begin{array}{l}\text { Influences activity by different susceptibility } \\
\text { to cleavage }[43,44]\end{array}$ \\
\hline \multirow[t]{2}{*}{ ICAM1 } & Arteriogenesis [23] & rs5498 & p.Lys469Glu & Exon 2 & Missense variant & 0.092 & - & $\begin{array}{l}\text { Related to coronary artery calcification } \\
\text { [52] }\end{array}$ & Related to s-ICAM1 levels [48-50] \\
\hline & & rs3093030 & c. $-286 C>T$ & $\begin{array}{l}\text { Non } \\
\text { coding } \\
\text { exon }\end{array}$ & $\begin{array}{l}\text { Non coding } \\
\text { transcript exon } \\
\text { variant }\end{array}$ & 0.208 & - & - & Related to s-ICAM1 levels $[49,51]$ \\
\hline TGFB1 & Both [17-19] & rs1800469 & c. $^{*} 309 \mathrm{~T}>\mathrm{C}$ & Promoter & $\begin{array}{l}\text { Regulatory region } \\
\text { variant }\end{array}$ & 0.208 & - & [53] & - \\
\hline CD53 & - & rs6679497 & $\begin{array}{l}\text { C.-17- } \\
5027 C>G\end{array}$ & Intron 2 & $\begin{array}{l}\text { Regulatory region } \\
\text { variant }\end{array}$ & & - & - & $\begin{array}{l}\text { Associated to TNFa levels [24], which has } \\
\text { been related to arteriogenesis [25] }\end{array}$ \\
\hline
\end{tabular}


Table 1 SNPs analyzed in the study (Continued)

POSTN Angiogenesis [26] $\quad$ rs3829365 $\quad$ c.-33C > G

Promoter Regulatory region 0

variant

rs1028728 C.-953 T>A Promoter Regulatory region 0.5

flanking variant

LGALS2 Both $[20,21]$

rs7291467 c.6+

Intron 1 Regulatory region

variant

Associated with heart failure [54]

Abbreviations: CCC, coronary collateral circulation; CAD, coronary artery disease. FS score: functional effects of SNPs obtained from 16 bioinformatics tools and databases. (http://compbio.cs.queensu.ca/F-SNP/) 
Table 2 Epidemiological and Clinical Characteristics of CAD patients with poor and good CCC

\begin{tabular}{llll}
\hline Characteristic & Poor CCC & Good CCC & p value \\
& $n=546(\%)$ & $n=131(\%)$ & \\
\hline Age (years) & $65.26 \pm 10.88$ & $66.76 \pm 10.06$ & 0.187 \\
Gender (male) & $460(84.25)$ & $110(83.97)$ & 0.937 \\
Hypertension (n) & $372(68.13)$ & $97(74.05)$ & 0.188 \\
Diabetes mellitus ( $n)$ & $146(26.74)$ & $47(35.88)$ & $0.037^{*}$ \\
Hyperlipidemia ( $n)$ & $381(69.78)$ & $96(73.28)$ & 0.430 \\
Smoking ( $n)$ & $126(23.08)$ & $33(25.19)$ & 0.608 \\
Angina history (n) & $383(70.15)$ & $93(70.99)$ & 0.849 \\
Previous myocardial infarction (n) & $196(35.90)$ & $43(32.82)$ & 0.509 \\
Medication with statins $(n)$ & $188(34.43)$ & $58(44.27)$ & $0.035^{*}$ \\
\hline
\end{tabular}

Abbreviations: CCC, coronary collateral circulation. Values are given as mean (S.D.) or numbers of patients (\%). $p<0.05$ was considered as statistically significant $\left({ }^{*}\right)$

POSTN haplotype analysis showed that the haplotype frequencies in patients with good collaterals were similar to those in patients with poor CCC (data not shown).

\section{Discussion}

An increasing number of SNPs are being accepted as underlying contributors to numerous cardiovascular disorders. Different researchers have shown the importance of several polymorphisms in CCC susceptibility [21, 28, 33, 46, 47]. In vitro studies have suggested that the p.Asp298Glu polymorphism plays a functional role, with the Asp 298 variant being associated with a decreased eNOS activity [43, 44], the consequences of which may include impaired collateral development. The Asp variant has been associated with poor CCC in 291 CAD patients with chronic coronary occlusions [45], and similar results have been reported in a series of 477 CAD patients with high-grade coronary stenosis $\geq 70 \%$ [46]. However, because NOS3 p.Asp298Glu deviates from HWE in our population, we could not analyze this polymorphism in our samples.

Another polymorphism which has been studied in relation to coronary arteriogenesis is p.Pro582Ser located in the HIF1A gene. The C/T polymorphism at nucleotide 85 of exon 12 results in a Pro/Ser polymorphism at residue 582 of HIF-1 $\alpha$. This substitution alters the amino acid sequence in the carboxyl-terminal domain of HIF- $1 \alpha$, which regulates protein stability and transcriptional activity [58]. Resar et al. demonstrated that CT or TT genotypes affecting residue 582 of the HIF- $1 \alpha$ protein were associated with the absence of coronary collaterals in 100 patients with CAD [27]. This result indicates that p.Pro582Ser substitution could influence the expression of angiogenic growth factors, thus leading to reduced collateral formation. Although we could not validate these results in our 677 CAD patients, our results are in agreement with those published by Alidoosti et al.
(2011) which found no association between rs11549465 variants and the extent of CCC $(n=196)$ [59]. Despite that study being conducted in Iranian CAD patients, our results support Alidoosti's observations, with our study being more robust based on a significantly higher number of patients $(n=677)$. Taking all this into account, the relevance of p.Pro582Ser HIF1A to CCC susceptibility is still under debate.

Unlike the results reported by Lin et al., 2010, showing that the VEGFA c. $-634 \mathrm{C}>\mathrm{G}(+405 \mathrm{C}>\mathrm{G}) \quad(\mathrm{rs} 2010963)$ and c. $2055 \mathrm{~A}>\mathrm{C}$ (A-2578C) (rs699947) polymorphisms were associated with the coronary arteriogenic response in 393 CAD patients [33], our results do not confirm the existence of any association between CCC and the allelic or genotypic distribution of this polymorphism. Given that the study by Lin et al. was conducted in Chinese patients, this discrepancy could be attributed to differences in population genetics.

Galectin-2, which is encoded by the LGALS2 gene, is an inhibitor of arteriogenesis [21]. This inhibition is dependent of the gene expression on the cell surface of monocytes, acting as a modulator of monocyte/macrophage responses during collateral artery growth. CAD patients with poor CCC have increased monocytic mRNA expression of galectin-2, independent of different stimulations of these cells. Interestingly, the mRNA expression of galectin-2 was significantly associated with the LGALS2 rs7291467 genotype, which has been associated with CCC in a small group of patients $(n=50)$ [21]. The same researchers also found that galectin- 2 was able to inhibit collateral circulation in a mouse model of limb ischemia [21]. However, we have being unable to demonstrate an association between arteriogenic response and the allelic or genotypic distribution of this polymorphism in our cohort of patients. This may be attributable to the fact that van der Laan's study used the collateral flow index as a quantitative measure of CCC, instead of poor and good CCC based on a qualitative angiographic Rentrop score.

The most extensively studied chemokine contributing to postischemic neovascularization is the monocyte chemo-attractant protein-1 (MCP-1); a protein which is overexpressed in collateral growth, allowing for monocyte recruitment sites [60]. The crucial role of monocytes in collateral growth is exemplified by the observations that genetic targeting of the MCP-1 gene (CCL2) and of the MCP-1 receptor gene (CCR2) leads to defective collateral growth [61, 62]. However, none of the SNPs of CCL2, rs2857654, rs1024611, rs1024610 and rs2857657, analyzed individually or their haplotype combinations were associated with CCC development.

The main limitation of the study is that the collateralization assessment is based on the angiographic Rentrop score, which is a qualitative rather than a quantitative 
Table 3 Association of genotype and allele distribution of examined polymorphisms with CAD patients with poor and good CCC

\begin{tabular}{|c|c|c|c|c|c|c|c|c|c|c|c|}
\hline \multirow{2}{*}{$\frac{\text { Gene }}{\text { VEGFA }}$} & \multirow{2}{*}{$\begin{array}{l}\mathrm{dbSNP} \text { ID } \\
\mathrm{rs} 2010963\end{array}$} & \multirow[t]{2}{*}{ Patients } & \multirow[t]{2}{*}{$\mathrm{n}$} & \multicolumn{3}{|c|}{ Genotype count (frequency) } & \multirow[t]{2}{*}{ P value ${ }^{a}$} & \multicolumn{2}{|c|}{ Allele count (frequency) } & \multirow[t]{2}{*}{$P$ value ${ }^{b}$} & \multirow[t]{2}{*}{ HWE P } \\
\hline & & & & GG & GC & $\mathrm{CC}$ & & G & C & & \\
\hline & & Poor CCC & 531 & $247(46.52)$ & $224(42.18)$ & $60(11.30)$ & 0.5760 & $718(67.61)$ & $344(32.39)$ & 0.495 & 0.8216 \\
\hline & & Good CCC & 121 & $50(41.32)$ & $58(47.93)$ & $13(10.75)$ & & $158(65.29)$ & $84(34.71)$ & & \\
\hline & rs1570360 & & & GG & GA & $\mathrm{AA}$ & & G & A & & \\
\hline & & Poor CCC & 451 & $207(45.90)$ & $197(43.68)$ & $47(10.42)$ & 0.782 & $611(67.74)$ & $291(32.26)$ & 0.521 & 0.8494 \\
\hline & & Good CCC & 97 & $47(48.45)$ & $42(43.30)$ & $8(8.25)$ & & $136(70.10)$ & $58(29.90)$ & & \\
\hline & rs699947 & & & CC & $A C$ & AA & & C & A & & \\
\hline & & Poor CCC & 494 & $138(27.94)$ & $245(49.59)$ & $111(22.47)$ & 0.816 & $521(52.73)$ & $467(47.27)$ & 0.968 & 0.5199 \\
\hline & & Good CCC & 104 & $31(29.81)$ & $48(46.15)$ & $25(24.04)$ & & $110(52.90)$ & $98(47.12)$ & & \\
\hline & rs3025039 & & & CC & $C T$ & $\pi$ & & C & $\mathrm{T}$ & & \\
\hline & & Poor CCC & 498 & $386(77.51)$ & $106(21.29)$ & $6(1.20)$ & 0.665 & 878 (88.15) & 118 (11.85) & 0.714 & 0.9533 \\
\hline & & Good CCC & 105 & $84(80)$ & 19 (18.10) & $2(1.90)$ & & 187 (89.05) & $23(10.95)$ & & \\
\hline & rs833061 & & & CC & $C T$ & $\pi$ & & C & $\mathrm{T}$ & & \\
\hline & & Poor CCC & 526 & $124(23.57)$ & $268(50.95)$ & $134(25.48)$ & 0.471 & $516(49.05)$ & $536(50.95)$ & 0.232 & 0.6392 \\
\hline & & Good CCC & 121 & $33(27.27)$ & $63(52.07)$ & $25(20.66)$ & & $129(53.31)$ & 113 (46.69) & & \\
\hline \multirow[t]{9}{*}{$\overline{K D R}$} & rs1870377 & & & $\pi$ & AT & $\mathrm{AA}$ & & $\mathrm{T}$ & A & & \\
\hline & & Poor CCC & 496 & 291 (58.67) & 178 (35.89) & $27(5.44)$ & 0.613 & 760 (76.61) & 232 (23.39) & 0.328 & 0.8991 \\
\hline & & Good CCC & 106 & $67(63.21)$ & 35 (33.02) & $4(3.77)$ & & 169 (79.72) & $43(20.28)$ & & \\
\hline & rs2305948 & & & CC & $\mathrm{CT}$ & $\pi$ & & C & $\mathrm{T}$ & & \\
\hline & & Poor CCC & 582 & 487 (83.68) & 88 (15.12) & $7(1.20)$ & 0.199 & $1062(91.24)$ & $102(8.76)$ & 0.207 & 0.3210 \\
\hline & & Good CCC & 153 & $120(78.43)$ & $32(20.92)$ & $1(0.65)$ & & 272 (88.89) & $34(11.11)$ & & \\
\hline & rs2071559 & & & $\pi$ & $C T$ & CC & & $\mathrm{T}$ & C & & \\
\hline & & Poor CCC & 544 & $147(27.02)$ & $276(50.74)$ & $121(22.24)$ & 0.319 & 570 (52.39) & $518(47.61)$ & 0.140 & 0.8355 \\
\hline & & Good CCC & 129 & 29 (22.48) & $64(49.61)$ & $36(27.91)$ & & $122(47.29)$ & $136(52.71)$ & & \\
\hline \multirow[t]{12}{*}{ CCL2 } & rs1024611 & & & $A A$ & $A G$ & GG & & A & G & & \\
\hline & & Poor CCC & 576 & $332(57.64)$ & $210(36.46)$ & $34(5.90)$ & 0.221 & $874(75.87)$ & $278(24.13)$ & 0.826 & 0.3186 \\
\hline & & Good CCC & 153 & 94 (61.44) & $46(30.06)$ & $13(8.50)$ & & $234(76.47)$ & $72(23.53)$ & & \\
\hline & rs1024610 & & & AA & AT & $\pi$ & & A & $\mathrm{T}$ & & \\
\hline & & Poor CCC & 516 & $312(60.47)$ & 180 (34.88) & $24(4.65)$ & 0.516 & 804 (77.91) & $228(22.09)$ & 0.715 & 0.6077 \\
\hline & & Good CCC & 112 & 68 (60.71) & $36(32.15)$ & $8(7.14)$ & & 172 (76.79) & $52(23.21)$ & & \\
\hline & rs2857657 & & & CC & CG & GG & & C & G & & \\
\hline & & Poor CCC & 511 & $309(60.47)$ & $181(35.42)$ & $21(4.11)$ & 0.365 & 799 (78.18) & $223(21.82)$ & 0.832 & 0.8093 \\
\hline & & Good CCC & 111 & 71 (63.96) & 33 (29.73) & $7(6.31)$ & & 175 (78.83) & $47(21.17)$ & & \\
\hline & rs2857654 & & & CC & $A C$ & AA & & C & A & & \\
\hline & & Poor CCC & 580 & $336(57.93)$ & $211(36.38)$ & $33(5.69)$ & 0.248 & $883(76.12)$ & $277(23.88)$ & 0.993 & 0.4284 \\
\hline & & Good CCC & 153 & 93 (60.78) & $47(30.72)$ & $13(8.50)$ & & $233(76.14)$ & $73(23.86)$ & & \\
\hline \multirow[t]{3}{*}{$\overline{\text { NOS3 }}$} & rs1799983 & & & GG & GT & $\pi$ & & G & $\mathrm{T}$ & & \\
\hline & & Poor CCC & 513 & $211(41.13)$ & $216(42.11)$ & $86(16.76)$ & 0.596 & 638 (62.18) & 388 (37.82) & 0.686 & $0.0157^{*}$ \\
\hline & & Good CCC & 110 & 46 (41.82) & $48(43.64)$ & $16(14.54)$ & & $140(63.64)$ & $80(36.36)$ & & \\
\hline \multirow[t]{5}{*}{ ICAM1 } & rs5498 & & & $A A$ & $A G$ & GG & & A & G & & \\
\hline & & Poor CCC & 516 & $136(26.36)$ & $246(47.67)$ & 134 (25.97) & 0.308 & $518(50.19)$ & $514(49.81)$ & 0.958 & 0.1039 \\
\hline & & Good CCC & 112 & $33(29.46)$ & $46(41.08)$ & $33(29.46)$ & & $112(50.00)$ & $112(50.00)$ & & \\
\hline & rs3093030 & & & CC & $\mathrm{CT}$ & $\pi$ & & C & $\mathrm{T}$ & & \\
\hline & & Poor CCC & 517 & 134 (25.92) & $248(47.97)$ & 135 (26.11) & 0.415 & $516(49.90)$ & $518(50.10)$ & 0.883 & 0.1535 \\
\hline
\end{tabular}


Table 3 Association of genotype and allele distribution of examined polymorphisms with CAD patients with poor and good CCC (Continued)

\begin{tabular}{|c|c|c|c|c|c|c|c|c|c|c|c|}
\hline & & Good CCC & 112 & $33(29.46)$ & $47(41.97)$ & $32(28.57)$ & & $113(50.45)$ & $111(49.55)$ & & \\
\hline \multirow[t]{3}{*}{ TGFB1 } & rs1800469 & & & GG & GA & $\mathrm{AA}$ & & G & A & & \\
\hline & & Poor CCC & 483 & $198(50.00)$ & $228(47.20)$ & $57(11.80)$ & 0.696 & $624(64.60)$ & $342(35.40)$ & 0.979 & 0.8844 \\
\hline & & Good CCC & 100 & $43(43.00)$ & $43(43.00)$ & $14(14.00)$ & & $129(64.50)$ & $71(35.50)$ & & \\
\hline \multirow[t]{3}{*}{$\overline{C D} 53$} & rs6679497 & & & GG & $\mathrm{GA}$ & $\mathrm{AA}$ & & $G$ & $A$ & & \\
\hline & & Poor CCC & 483 & $198(41.00)$ & $228(47.20)$ & $57(11.80)$ & 0.826 & $624(64.60)$ & $342(35.40)$ & 0.572 & 0.6712 \\
\hline & & Good CCC & 100 & $43(43.00)$ & $43(43.00)$ & $14(14.00)$ & & $129(64.50)$ & $71(35.50)$ & & \\
\hline \multirow[t]{6}{*}{ POSTN } & rs3829365 & & & GG & GC & CC & & $G$ & $C$ & & \\
\hline & & Poor CCC & 405 & 357 (88.15) & $22(5.43)$ & $26(6.42)$ & 0.795 & 736 (90.86) & $74(9.14)$ & 0.535 & $0.0000^{*}$ \\
\hline & & Good CCC & 76 & 69 (90.79) & $3(3.95)$ & $4(5.26)$ & & 141 (92.76) & $11(7.24)$ & & \\
\hline & rs1028728 & & & AA & AT & $\pi$ & & A & $\mathrm{T}$ & & \\
\hline & & Poor CCC & 389 & $242(62.21)$ & $128(32.91)$ & $19(4.88)$ & 0.230 & 612 (78.66) & $166(21.34)$ & 0.105 & 0.7373 \\
\hline & & Good CCC & 77 & $54(70.13)$ & $22(28.57)$ & $1(1.30)$ & & $130(84.42)$ & $24(15.58)$ & & \\
\hline \multirow[t]{3}{*}{ LGALS2 } & rs7291467 & & & AA & $A G$ & GG & & $A$ & $G$ & & \\
\hline & & Poor CCC & 581 & $160(27.54)$ & $292(50.26)$ & $129(22.20)$ & 0.106 & $612(52.67)$ & $550(47.33)$ & 0.080 & 0.9589 \\
\hline & & Good CCC & 151 & $37(24.50)$ & $68(45.03)$ & $46(30.47)$ & & $142(47.02)$ & 160 (52.98) & & \\
\hline \multirow[t]{6}{*}{ HIF1A } & rs11549465 & & & $\mathrm{CC}$ & CT & $\pi$ & & $C$ & $\mathrm{~T}$ & & \\
\hline & & Poor CCC & 518 & $402(77.60)$ & $111(21.43)$ & $5(0.97)$ & 0.563 & 915 (88.32) & 121 (11.68) & 0.474 & 0.4122 \\
\hline & & Good CCC & 112 & $84(75)$ & $26(23.21)$ & $2(1.79)$ & & 194 (86.61) & 30 (13.39) & & \\
\hline & rs2057482 & & & $\mathrm{CC}$ & CT & $\pi$ & & C & $\mathrm{T}$ & & \\
\hline & & Poor CCC & 497 & $339(68.21)$ & $148(29.78)$ & $10(2.01)$ & 0.490 & $826(83.10)$ & 168 (16.90) & 0.328 & 0.1151 \\
\hline & & Good CCC & 111 & $70(63.06)$ & 38 (34.24) & $3(2.70)$ & & $178(80.18)$ & 44 (19.82) & & \\
\hline
\end{tabular}

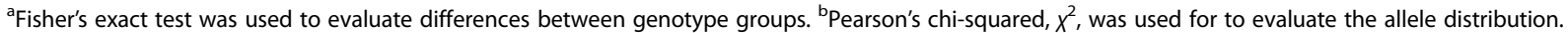
${ }^{*} \mathrm{p}<0.05$ was considered as statistically significant

technique. A modified Rentrop method without occlusion of the recipient artery was performed in the current work. This method, as well as the inclusion of a large portion of patients with subocclusive lesions (>70-100 \%), probably might explain why such a relative low number of patients displayed well-developed collateral arteries in this cohort. Also, functional polymorphims in interferonbeta signaling genes, which are involved in arteriogenesis from clinical studies [63, 64], were not included in the study.

\section{Conclusions}

Despite having previously reported that PLAU p.Pro141Leu (rs2227564) was associated with coronary arteriogenesis [5], none of the rs11549465, rs2057482, rs2010963, rs1570360, rs699947, rs3025039, rs833061, rs1870377, rs2305948, rs2071559, rs1024611, rs1024610, rs2857657, rs2857654, rs1799983, rs5498, rs3093030, rs1800469, rs6679497, rs3829365 or rs1028728 polymorphisms analyzed located in or close to genes involved in postischemic neovascularization (VEGFA, KDR, CCL2, ICAM1 and $P O S T N$ ) or their haplotype combinations were associated with CCC development. In addition, in our cohort of patients we could not validate the association of the NOS3 rs1799983, HIF1A rs11549465, VEGFA rs2010963 and rs699947, and LGALS2 rs7291467 polymorphisms with CCC development reported by other authors. We and others have demonstrated the potential role of certain polymorphisms as factors associated with CCC $[5,21,28$, $45,46]$, but usually they have not been validated in other cohorts of patients. In addition, SNPs may influence collateral development not only individually, but also when acting together with other SNPs, through gene haplotype networks, as demonstrated by the role of several inflammatory gene haplotype networks in CCC [65]. In conclusion, a validated SNP-based GWAS is needed to reveal and/or confirm the SNPs that predict coronary arteriogenic response.

\section{Competing interests}

The author(s) declare that they have no competing interests.

\section{Authors' contributions}

JA and MP were responsible for study conception and design. JA and JD analyzed and interpreted the study data, and wrote the first draft of the manuscript. JD, MM, VG, AC and OA were responsible for clinical and 
epidemiological data collection. JD, MM and AC for DNA genotyping. JC, EM, $\mathrm{OA}, \mathrm{IO}$ and $\mathrm{BG}$ were responsible for measurements of angiographic parameters. $P S, M M$, and JD were responsible for statistical analysis. JA, JD, VG, DG, BG and $J R$ assisted in the acquisition of data and provided valuable, intellectual contributions to the manuscript draft. All authors have approved the final version of the manuscript.

\section{Acknowledgements}

We thank Montse Cairó, Eva Sánchez, Dolors Colell, Teresa Torrent, María José Fernández de Muniain, and Miquel Rugat for their valuable collaboration and technical assistance. This study was supported by the Fundació La Marató de TV3 07 (project 080810) and the Universitat de Barcelona (project ACESBELL 08).

\section{Author details \\ ${ }^{1}$ Unitat d'Anatomia i Embriologia Humanes, Departament de Patologia i Terapèutica Experimental, Facultat de Medicina, Campus de Ciències de la Salut de Bellvitge, Universitat de Barcelona, L'Hospitalet de Llobregat, Barcelona 08907, Spain. ${ }^{2}$ Departamento de Estadística, Facultad de Ciencias, Universidad de Valparaíso, Valparaíso, Chile. ${ }^{3}$ Neurovascular Research Group (NEUVAS), Institut Municipal d'Investigació Mèdica. Hospital del Mar, PRBB, Parc de Recerca Biomèdica de Barcelona, Barcelona, Spain. ${ }^{4}$ Department of Cardiology and Hemodynamics, Centre Cardiovascular Sant Jordi, Barcelona, Spain. ${ }^{5}$ Department of Cardiology, Hospital Universitari Vall d'Hebron, Barcelona, Spain. ${ }^{6}$ Department of Cardiology, Hospital Mútua de Terrassa, Terrassa, Barcelona, Spain. ${ }^{7}$ Departament of Morphological Sciences, Universitat Autònoma de Barcelona, Bellaterra, Cerdanyola del Vallès, Barcelona, Spain. ${ }^{8}$ Àrea Epigenetics and Cancer Biology Area, Institut d'Investigació Biomèdica de Bellvitge (IDIBELL), L'Hospitalet de Llobregat, Barcelona, Spain.}

\section{Received: 10 December 2014 Accepted: 21 April 2015 Published online: 12 May 2015}

\section{References}

1. Meier P, Hemingway $H$, Lansky AJ, Knapp G, Pitt B, Seiler C. The impact of the coronary collateral circulation on mortality: a meta-analysis. Eur Heart J. 2012;33(5):614-21.

2. Pohl T, Seiler C, Billinger M, Herren E, Wustmann $K$, Mehta $H$, et al. Frequency distribution of collateral flow and factors influencing collateral channel development. Functional collateral channel measurement in 450 patients with coronary artery disease. J Am Coll Cardiol. 2001;38(7):1872-8.

3. Schirmer SH, van Nooijen FC, Piek JJ, van Royen N. Stimulation of collatera artery growth: travelling further down the road to clinical application. Heart. 2009;95(3):191-7.

4. Silvestre JS, Mallat Z, Tedgui A, Levy BI. Post-ischaemic neovascularization and inflammation. Cardiovasc Res. 2008;78(2):242-9.

5. Duran J, Sánchez-Olavarría P, Mola M, Götzens V, Carballo J, Martín-Pelegrina E, et al. The PLAU P141L single nucleotide polymorphism is associated with collateral circulation in patients with coronary artery disease. Rev Esp Cardiol (Engl Ed). 2014;67(7):552-7.

6. Rentrop KP, Cohen M, Blanke H, Phillips RA. Changes in collateral channel filling immediately after controlled coronary artery occlusion by an angioplasty balloon in human subjects. J Am Coll Cardiol. 1985;5(3):587-92.

7. Coulon C, Georgiadou M, Roncal C, De Bock K, Langenberg T, Carmeliet P. From vessel sprouting to normalization: role of the prolyl hydroxylase domain protein/hypoxia-inducible factor oxygen-sensing machinery. Arterioscler, Thromb, Vasc Biol. 2010;30(12):2331-6.

8. Patel TH, Kimura H, Weiss CR, Semenza GL, Hofmann LV. Constitutively active HIF-1a improves perfusion and arterial remodeling in an endovascular model of limb ischemia. Cardiovasc Res. 2005;68(1):144-54.

9. Duran J, Götzens V, Carballo J, Martn E, Petit M, Cordero Á, et al. The HIF1A C85T single nucleotide polymorphism influences the number of branches of the human coronary tree. Cardiology. 2012;121(3):156-9.

10. Hoeben A, Landuyt B, Highley MS, Wildiers H, Van Oosterom AT, De Bruijn EA. Vascular endothelial growth factor and angiogenesis. Pharmacol Rev. 2004:56(4):549-80.

11. Clayton JA, Chalothorn D, Faber JE. Vascular endothelial growth factor-a specifies formation of native collaterals and regulates collateral growth in ischemia. Circ Res. 2008;103(9):1027-36.
12. Toyota E, Warltier DC, Brock T, Ritman E, Kolz C, O'Malley P, et al. Vascular endothelial growth factor is required for coronary collateral growth in the rat. Circulation. 2005;112(14):2108-13.

13. Hsu HW, Wall NR, Hsueh CT, Kim S, Ferris RL, Chen CS, et al. Combination antiangiogenic therapy and radiation in head and neck cancers. Oral Oncol. 2014;50(1):19-26.

14. Babiak A, Schumm AM, Wangler C, Loukas M, Wu J, Dombrowski S, et al. Coordinated activation of VEGFR-1 and VEGFR-2 is a potent arteriogenic stimulus leading to enhancement of regional perfusion. Cardiovasc Res. 2004;61(4):789-95.

15. Cooke JP, Ghebremariam YT. Endothelial nicotinic acetylcholine receptors and angiogenesis. Trends Cardiovasc Med. 2008;18(7):247-53.

16. Schaper W. Collateral circulation: past and present. Basic Res Cardiol. 2009;104(1):5-21.

17. van Royen N, Hoefer I, Buschmann I, Heil M, Kostin S, Deindl E, et al. Exogenous application of transforming growth factor beta 1 stimulates arteriogenesis in the peripheral circulation. FASEB J. 2002;16(3):432-4.

18. Peshavariya HM, Chan EC, Liu GS, Jiang F, Dusting GJ. Transforming growth factor- $\beta 1$ requires NADPH oxidase 4 for angiogenesis in vitro and in vivo. J Cell Mol Med. 2014;18(6):1172-83.

19. Grundmann S, van Royen N, Pasterkamp G, Gonzalez N, Tijsma EJ, Piek JJ, et al. A New intra-arterial DeliveryPlatform for Pro-arteriogenic compounds to stimulate collateral artery growth Via transforming growth factor-beta1 release. J Am Coll Cardiol. 2007:50(4):351-8.

20. Chen C, Duckworth CA, Fu B, Pritchard DM, Rhodes JM, Yu L-G. Circulating galectins $-2,-4$ and -8 in cancer patients make important contributions to the increased circulation of several cytokines and chemokines that promote angiogenesis and metastasis. Br J Cancer. 2014;110(3):741-52.

21. Van der Laan AM, Schirmer SH, de Vries MR, Koning JJ, Volger OL, Fledderus JO, et al. Galectin-2 expression is dependent on the rs7291467 polymorphism and acts as an inhibitor of arteriogenesis. Eur Heart J. 2011;33(9):1076-84.

22. Keeley EC, Mehrad B, Strieter RM. Chemokines as mediators of neovascularization. Arterioscler, Thromb, Vasc Biol. 2008;28(11):1928-36.

23. Hoefer IE, van Royen N, Rectenwald JE, Deindl E, Hua J, Jost M, et al. Arteriogenesis proceeds via ICAM-1/Mac-1- mediated mechanisms. Circ Res. 2004;94(9):1179-85.

24. Bos SD, Lakenberg N, van der Breggen R, Houwing-Duistermaat JJ, Kloppenburg $\mathrm{M}$, de Craen AJ, et al. A genome-wide linkage scan reveals CD53 as an important regulator of innate TNF-alpha levels. Eur J Hum Genet. 2010;18(8):953-9.

25. Hoefer IE, van Royen N, Rectenwald JE, Bray EJ, Abouhamze Z, Moldawer LL, et al. Direct evidence for tumor necrosis factor-alpha signaling in arteriogenesis. Circulation. 2002:105(14):1639-41.

26. Shao R, Bao S, Bai X, Blanchette C, Anderson RM, Dang T, et al. Acquired expression of periostin by human breast cancers promotes tumor angiogenesis through up-regulation of vascular endothelial growth factor receptor 2 expression. Mol Cell Biol. 2004;24(9):3992-4003.

27. Hlatky MA, Quertermous T, Boothroyd DB, Priest JR, Glassford AJ, Myers RM, et al. Polymorphisms in hypoxia inducible factor 1 and the initial clinical presentation of coronary disease. Am Heart J. 2007;154(6):1035-42.

28. Resar JR, Roguin A, Voner J, Nasir K, Hennebry TA, Miller JM, et al. Hypoxia-inducible factor 1alpha polymorphism and coronary collaterals in patients with ischemic heart disease. Chest. 2005;128(2):787-91.

29. Watson C, Webb NJ, Bottomley MJ, Brenchley PE. Identification of polymorphisms within the vascular endothelial growth factor (VEGF) gene: correlation with variation in VEGF protein production. Cytokine+. 2000;12(8):1232-5.

30. Steffensen KD, Waldstrøm M, Brandslund I, Jakobsen A. The relationship of VEGF polymorphisms with serum VEGF levels and progression-free survival in patients with epithelial ovarian cancer. Gynecol Oncol. 2010;117(1):109-16.

31. Mohammadi M, Bazrafshani MR, Day PJ, Ollier WE. Vascular endothelial growth factor production is regulated by gene polymorphisms. Iran J Immunol. 2009;6(3):119-29.

32. Al-Habboubi HH, Sater MS, Almawi AW, Al-Khateeb GM, Almawi WY. Contribution of VEGF polymorphisms to variation in VEGF serum levels in a healthy population. Eur Cytokine Netw. 2011;22(3):154-8.

33. Lin TH, Wang CL, Su HM, Hsu PC, Juo SH, Voon WC, et al. Functional vascular endothelial growth factor gene polymorphisms and diabetes: effect on coronary collaterals in patients with significant coronary artery disease. Clin Chim Acta. 2010;411(21-22):1688-93.

34. Cui QT, Li Y, Duan CH, Zhang W, Guo XL. Further evidence for the contribution of the vascular endothelial growth factor gene in coronary artery disease susceptibility. Gene. 2013;521(2):217-21. 
35. Wang $Y$, Zheng $Y$, Zhang W, Yu H, Lou K, Zhang Y, et al. Polymorphisms of KDR gene are associated with coronary heart disease. J Am Coll Cardiol. 2007:50(8):760-7.

36. Rovin BH, Lu L, Saxena R. A novel polymorphism in the MCP-1 gene regulatory region that influences MCP-1 expression. Biochem Biophys Res Commun. 1999;259(2):344-8.

37. Del Guerra S, D'Aleo V, Gualtierotti G, Filipponi F, Boggi U, De Simone P, et al. A common polymorphism in the monocyte chemoattractant protein-1 (MCP-1) gene regulatory region influences MCP-1 expression and function of isolated human pancreatic islets. Transpl Proc. 2010;42(6):2247-9.

38. Pham MHT, Bonello GB, Castiblanco J, Le T, Sigala J, He W, et al. The rs 1024611 regulatory region polymorphism is associated with CCL2 allelic expression imbalance. PLoS One. 2012;7(11):e49498.

39. McDermott DH, Yang Q, Kathiresan S, Cupples LA, Massaro JM, Keaney Jr JF, et al. CCL2 polymorphisms are associated with serum monocyte chemoattractant protein-1 levels and myocardial infarction in the Framingham Heart Study. Circulation. 2005;112(8):1113-20.

40. Tabara Y, Kohara K, Yamamoto Y, Igase M, Nakura J, Kondo I, et al. Polymorphism of the monocyte chemoattractant protein (MCP-1) gene is associated with the plasma level of MCP-1 but not with carotid intima-media thickness. Hypertens Res. 2003;26(9):677-83.

41. Gonzalez E, Rovin BH, Sen L, Cooke G, Dhanda R, Mummidi S, et al. HIV-1 infection and AIDS dementia are influenced by a mutant MCP-1 allele linked to increased monocyte infiltration of tissues and MCP-1 levels. Proc Natl Acad Sci U S A. 2002;99(21):13795-800.

42. Lin HL, Ueng KC, Hsieh YS, Chiang WL, Yang SF, Chu SC. Impact of MCP-1 and CCR-2 gene polymorphisms on coronary artery disease susceptibility. Mol Biol Rep. 2012;39(9):9023-30.

43. Tesauro M, Thompson WC, Rogliani P, Qi L, Chaudhary PP, Moss J. Intracellular processing of endothelial nitric oxide synthase isoforms associated with differences in severity of cardiopulmonary diseases: cleavage of proteins with aspartate vs. glutamate at position 298. Proc Natl Acad Sci U S A. 2000;97(6):2832-5.

44. Persu A, Stoenoiu MS, Messiaen T, Davila S, Robino C, El-Khattabi O, et al. Modifier effect of ENOS in autosomal dominant polycystic kidney disease. Hum Mol Genet. 2002;11(3):229-41.

45. Lamblin N, Cuilleret FJ, Helbecque N, Dallongeville J, Lablanche J-M, Amouyel $\mathrm{P}$, et al. A common variant of endothelial nitric oxide synthase (Glu298Asp) is associated with collateral development in patients with chronic coronary occlusions. BMC Cardiovasc Disord. 2005;5:27.

46. Gulec S, Karabulut H, Ozdemir AO, Ozdol C, Turhan S, Altin T, et al. Glu298Asp polymorphism of the eNOS gene is associated with coronary collateral development. Atherosclerosis. 2008;198(2):354-9.

47. Abdel-Aziz TA, Mohamed RH. Association of endothelial nitric oxide synthase gene polymorphisms with classical risk factors in development of premature coronary artery disease. Mol Biol Rep. 2013;40(4):3065-71.

48. Bielinski SJ, Pankow JS, Li N, Hsu FC, Adar SD, Jenny NS, et al. ICAM1 and VCAM1 polymorphisms, coronary artery calcium, and circulating levels of soluble ICAM-1: the multi-ethnic study of atherosclerosis (MESA). Atherosclerosis. 2008;201(2):339-44.

49. Bielinski SJ, Pankow JS, Foster CL, Miller MB, Hopkins PN, Eckfeldt JH, et al. Circulating soluble ICAM-1 levels shows linkage to ICAM gene cluster region on chromosome 19: The NHLBI Family Heart Study follow-up examination. Atherosclerosis. 2008;199(1):172-8.

50. Bielinski SJ, Reiner AP, Nickerson D, Carlson C, Bailey KR, Thyagarajan B, et al. Polymorphisms in the ICAM1 gene predict circulating soluble intercellular adhesion molecule-1(sICAM-1). Atherosclerosis. 2011;216(2):390-4.

51. Barbalic M, Dupuis J, Dehghan A, Bis JC, Hoogeveen RC, Schnabel RB, et al. Large-scale genomic studies reveal central role of $\mathrm{ABO}$ in $\mathrm{SP}$-selectin and sICAM-1 levels. Hum Mol Genet. 2010;19(9):1863-72.

52. Reilly MP, Wolfe ML, Dykhouse J, Reddy K, Localio AR, Rader DJ. Intercellular adhesion molecule 1 (ICAM-1) gene variant is associated with coronary artery calcification independent of soluble ICAM-1 levels. J Investig Med. 2004;52(8):515-22.

53. Morris DR, Moxon JV, Biros E, Krishna SM, Golledge J. Meta-analysis of the association between transforming growth Factor-Beta polymorphisms and complications of coronary heart disease. PLoS One. 2012;7(5):e37878.

54. Wang F, Song Y, Jiang Y, Yang C, Ding Z. Associations among periostin gene polymorphisms, clinical parameters and heart failure: a case-control study in 1104 Chinese individuals. J Cardiovasc Med (Hagerstown). 2011;12(7):469-74.

55. Ozaki K, Inoue $K$, Sato $H$, lida A, Ohnishi Y, Sekine A, et al. Functional variation in LGALS2 confers risk of myocardial infarction and regulates lymphotoxin-alpha secretion in vitro. Nature. 2004;429(6987):72-5.

56. Ozaki K, Tanaka T. Genome-wide association study to identify SNPs conferring risk of myocardial infarction and their functional analyses. Cell Mol Life Sci. 2005;62(16):1804-13.

57. Asselbergs FW, Pai JK, Rexrode KM, Hunter DJ, Rimm EB. Effects of lymphotoxin-alpha gene and galectin-2 gene polymorphisms on inflammatory biomarkers, cellular adhesion molecules and risk of coronary heart disease. Clin Sci (Lond). 2007;112(5):291-8.

58. Tanimoto K, Yoshiga K, Eguchi H, Kaneyasu M, Ukon K, Kumazaki T, et al. Hypoxia-inducible factor-1alpha polymorphisms associated with enhanced transactivation capacity, implying clinical significance. Carcinogenesis. 2003;24(11):1779-83.

59. Alidoosti M, Ghaedi M, Soleimani A, Bakhtiyari S, Rezvanfard M, Golkhu S, et al. Study on the role of environmental parameters and HIF-1A gene polymorphism in coronary collateral formation among patients with ischemic heart disease. Clin Biochem. 2011;44(17-18):1421-4.

60. Ito WD, Arras M, Winkler B, Scholz D, Schaper J, Schaper W. Monocyte chemotactic protein-1 increases collateral and peripheral conductance after femoral artery occlusion. Circ Res. 1997;80(6):829-37.

61. Heil M, Ziegelhoeffer T, Wagner S, Fernandez B, Helisch A, Martin S, et al. Collateral artery growth (arteriogenesis) after experimental arterial occlusion is impaired in mice lacking CC-chemokine receptor-2. Circ Res. 2004;94(5):671-7.

62. Voskuil M, Hoefer IE, van Royen N, Hua J, de Graaf S, Bode C, et al. Abnormal monocyte recruitment and collateral artery formation in monocyte chemoattractant protein-1 deficient mice. Vasc Med. 2004;9(4):287-92.

63. Schirmer SH, Bot PT, Fledderus JO, van der Laan AM, Volger OL, Laufs U, et al. Blocking interferon \{beta\} stimulates vascular smooth muscle cell proliferation and arteriogenesis. J Biol Chem. 2010;285(45):34677-85.

64. Schirmer SH, Fledderus JO, Bot PT, Moerland PD, Hoefer IE, Baan Jr J, et al. Interferon-beta signaling is enhanced in patients with insufficient coronary collateral artery development and inhibits arteriogenesis in mice. Circ Res. 2008;102(10):1286-94.

65. Zhang J, Regieli JJ, Schipper M, Entius MM, Liang F, Koerselman J, et al. Inflammatory gene haplotype-interaction networks involved in coronary collateral formation. Hum Hered. 2008;66(4):252-64.

\section{Submit your next manuscript to BioMed Central and take full advantage of:}

- Convenient online submission

- Thorough peer review

- No space constraints or color figure charges

- Immediate publication on acceptance

- Inclusion in PubMed, CAS, Scopus and Google Scholar

- Research which is freely available for redistribution

Submit your manuscript at www.biomedcentral.com/submit
C BioMed Central 